\title{
Intense laser matter interaction in atoms, finite systems and condensed media: recent experiments and theoretical advances
}

\author{
Sivarama Krishnan ${ }^{1, a}$ and Marcel Mudrich²,3,b \\ 1 QuCenDiEM-group and Department of Physics, Indian Institute of Technology Madras, Chennai 600036, India \\ 2 Department of Physics and Astronomy, Aarhus University, 8000 Aarhus C, Denmark \\ 3 Department of Physics, Indian Institute of Technology Madras, Chennai 600036, India
}

Published online 5 January 2022

(C) The Author(s), under exclusive licence to EDP Sciences, Springer-Verlag GmbH Germany, part of Springer Nature 2021

\begin{abstract}
This special issue of the European Journal of Physics: Special Topics entitled "Intense Laser Matter Interaction in Atoms, Finite Systems and Condensed Media" published a set of 21 articles aiming to put in perspective this burgeoning area of science, application and technology. The invention of chirped pulse amplification by Strickland and Mourou, which led to their Nobel prizes in 2018, has ushered in a new era in photon-matter interaction where coherent intense light pulses in the optical and infrared domains play a central role. This development has not only called in creative experimental methods, but has demanded new theoretical approaches working in the non-perturbative and highly nonlinear regimes of light-matter interaction. Both for fundamental physics as well as key applications these have played an essential role. Key pillars of this subject, captured in this collection, are laser-induced nanometerscale plasmas ignited in unsupported nanoparticles, laser-based particle acceleration taking advantage of unprecedented field gradients, the generation of high-order harmonics opening up attosecond science and ultrafast X-ray spectroscopy, and the generation of terahertz pulses for time-domain spectroscopy of new materials.
\end{abstract}

\section{Introduction}

Laser-matter interaction is an important area of physics explored with equal enthusiasm both in experiments and theory. It encompasses a wide range of topics in research which are both of fundamental importance as well as cross-disciplinary application. This involves a whole range of interactions between light and matter from single and entangled photons [1] to classical waves obeying Maxwell's equations going up to very intense fields [2,3] reaching the Schwinger limit [4]. At every stage of these interactions a new level of complexity [5] is introduced leading to the development of a substantial body of knowledge which often involves one or more Nobel prizes [6]. Therefore, we prudently restrict this issue to important aspects of intense laser-matter interaction albeit in a wide range of media-atoms, finite systems and the condensed phase. However, this is not entirely deliberate as this special issue is inspired by the award of the Nobel Prize in 2018 to Arthur Ashkin, Gérard Mourou and Donna Strickland "for groundbreaking inventions in the field of laser physics" [7].

The advent of ultrashort laser-pulse amplification has opened the way to studying light-matter interaction in

\footnotetext{
a e-mail: srkrishnan@iitm.ac.in

b e-mail: mudrich@phys.au.dk (corresponding author)
}

new regimes. At intensities where the strength of the light field approaches or even surpasses the internal electric field inside atoms and solid bodies, the response of matter to light is dominated by nonlinear phenomena such as white-light generation [8], filament propagation [9], and the generation of high-order harmonics and attosecond pulses [10]. By strong-field ionization, condensed media are transformed into plasmas that feature drastically altered optical properties. A great challenge for current and future research is to deepen our understanding of laser-induced plasmas and, based on that, to develop strategies to utilize them for applications such as extreme-ultraviolet photolithography [11], micromachining [12], biomedical imaging [13], or particle acceleration [14].

This special issue features 21 articles addressing some of these and other related current research topics both from an experimental and a theoretical perspective. These are due to the efforts of leading and upcoming researchers in various aspects of this rich field of work including theory, computation and experiment. The wide span of geographies of the contributing authors is another testimony to the immense importance of this vista of science captured in this issue.

This collection contains articles on the theoretical aspects laying the foundation for intense lasermatter interaction and its immediate consequences such 
as high-order harmonic generation (HHG) and laserinduced plasmas. The recent application of HHG to investigate solids and nanostructures is covered by representative recent research published in this issue through contributions detailing ongoing theoretical as well as experimental efforts. An important contribution to this issue presents the details of the multi-purpose beamline at the Extreme Light Infrastructure facility which enables and advances this science. Attosecond pulses resulting from these coherent sets of harmonics has revolutionized ultrafast dynamics pushing the envelope down to sub-100 as. This enables probing tiny delays in photoemission processes predicted several decades ago by Wigner [15] — an article each on the theoretical and experimental aspects of related physics are captured in this issue. Laser-induced plasmas have received special attention owing to the unique opportunity presented by the confinement of these highlycharged media. Paradigmatic systems - doped helium nanodroplets exposed to intense soft X-ray pulses, as well as non-uniform charging and resonance absorption in fusion-ready nanoplasmas induced by intense nearIR pulses are presented by constituting authors of this issue. Further, analytical studies on exploiting the chirp of fs pulses for particle acceleration and exact solutions for charged particle motion in intense fields are discussed. This collection also hosts a few articles which are related to the central theme but may be considered off-mainstream. A tutorial review presents the physics of realizing strong light-matter coupling in the context of polaritons - a topic of growing interest in condensedmatter research. Further, terahertz pulses originating primarily from intense laser-matter interaction albeit in the perturbative regime are the subject of discussion in a couple of articles.

Thus, this issue serves the original intent of being a journey from the foundations and fundamentals of the science of intense laser-matter interactions through to the state-of-the-art in physics, applications as well as impact in related areas. This effort has been greatly facilitated by the guest editors' long-standing and fruitful collaboration currently supported by the Scheme for Promotion of Academic and Research Collaboration (SPARC), established by the Ministry of Human Resource Development, Government of India.

\section{Laser-induced nanoplasmas}

Free atomic clusters and nanoparticles are particularly attractive targets to study light-matter interaction in regimes of high intensity as provided by amplified ultrashort laser pulses. Nanoclusters exposed to intense pulses tend to undergo avalanche ionization resulting in Coulomb explosion of the system on the time scale of $0.1-10 \mathrm{ps}$. The final products are hot, quasi-thermal electrons, multiply charged energetic ions, and visible up to X-ray photons $[16,17]$. The most interesting properties of clusters driven by infrared pulses result from their high energy absorp- tion per atom once ionized, by far exceeding the values achievable in atomic jets or planar solid targets. The underlying mechanism is resonance absorption by an electronic nanoplasma which forms through avalanche ionization. The resonance occurs when the nanoplasma eigenfrequency matches the frequency of the laser light [18]. For these fundamental studies, rare-gas clusters are the preferred target systems because they represent dense assemblies of atoms that are nearly unperturbed by chemical bonds and because they are relatively easy to produce in free jets. While laser-induced nanoplasmas are fairly well understood owing to dedicated experiments and detailed model calculations, certain details of the complex ionization dynamics remain unresolved. These include the coupling between electrons and ions through collisions, the charge migration within the nanoplasma, and the recombination of electrons and ions into highly excited atoms and ions. Multi-component clusters present a particularly rich variety of energy and charge transfer and ionization processes. In particular doped helium nanodroplets have attracted considerable interest in this context due to their superfluid nature, the extreme difference between ionization energies of guest species and the host substance (helium), and the high level of control of the structure of the dopant-droplet system [19-22].

In this special issue, intense laser-matter interaction is studied experimentally by Komar and coworkers using free argon clusters at laser intensities up to $10^{15} \mathrm{Wcm}^{-2}[23]$. Using a charge-state resolving ion energy analyzer based on a homogeneous magnetic field and position as well as time-of-flight sensitive detection, the recoil ion energy distributions were measured for charge states $\mathrm{Ar}^{+}$up to $\mathrm{Ar}^{8+}$. These were well described by a model which takes into account saturation of ionization as well as details of the cluster size and laser intensity distributions. At lower laser intensities, the ion energy spectra revealed distinct low-energy gaps for the higher charge states, pointing at three-body recombination occurring already during the laser pulse. The authors concluded that the charge-state distributions developing during the laser pulse are inhomogeneous.

Jha and coworkers also present experimental studies on the ionization of argon clusters, though at higher laser intensities up to $4 \times 10^{18} \mathrm{Wcm}^{-2}$ [24]. Using a Thomson parabola ion spectrometer, they were able to measure ion charge-state propensity distributions and charge-resolved kinetic energy spectra for Coulombexploded argon clusters. For the most abundant ion, $\mathrm{Ar}^{8+}$, kinetic energies up to $200 \mathrm{keV}$ were measured. To fit ion energy spectra, the authors extend a previous model based on uniform charging of the clusters and propose a non-uniform charging scheme, similarly to the work by Komar et al. [23]. This scheme gives detailed insight into location of ions of different charges, their energies, as well as the extent of screening by the quasifree electrons generated from the inner-ionization.

In an international collaboration lead by Möller, Neumark, Bostedt, Vilesov and Gessner, Saladrigas studied the ejection of ions from charged helium nanodroplets 
ionized by intense femtosecond X-ray laser pulses by ion time-of-flight (TOF) spectroscopy in coincidence with small-angle X-ray scattering [25]. The advantage of the single-particle X-ray-imaging technique is that the size of the target particle and the absolute photon flux incident on each particle can be determined, while ion TOF spectra inform about the maximum ion kinetic energy. Owing to the strongly fluctuating experimental conditions in this event-by-event detection scheme, a wide variety of regimes of ionization and expansion are probed, spanning from the departure of all photoelectrons from the droplet, leading to pure Coulomb explosion, to substantial electron trapping by the electrostatic potential of the charged droplet, indicating the onset of hydrodynamic expansion. The findings are consistent with the emergence of a charged spherical shell around a quasineutral plasma. The results demonstrate a complex relationship between measured ion kinetic energy and droplet ionization conditions.

In their theoretical work using molecular dynamics (MD) simulations, Mahalik and Kundu studied the absorption resonance in a laser-driven deuterium cluster as a function of the intensity, polarization, and duration of the laser pulse [26]. They find that the resonance shifts to lower frequencies for increasing laser intensity and disappears at intensities where ionization saturates. For intensities $<10^{17} \mathrm{~W} \mathrm{~cm}^{-2}$, the optimum wavelength for maximum light absorption in deuterium clusters lies around $\lambda=330 \mathrm{~nm}$.

Last, Jortner and Heidenreich studied the energy exchange between electrons and ions in laser-ionized, exploding clusters on a microscopic level using MD simulations [27]. A snapshot of an exploding neon cluster is shown in Fig. 1. For the system's electrons and $\left(\mathrm{H}^{+}\right)_{N}$ ions as well as electrons and $\left(\mathrm{Ne}^{8+}\right)_{N}$ ions, they compute electron-ion collision times ranging between 10 and 50 as. When considering collisional energy transfer within the entire cluster, electron-to-ion and ion-toelectron energy transfer was found to be close to balance, providing on average a low net energy transfer to ions. These findings provide strong evidence for the absence of the so-called hydrodynamic expansions and for the dominance of Coulomb interactions in the explosion of the cluster nanoplasmas.

In another theoretical work based on MD simulations, Heidenreich and Mudrich investigated the explosion dynamics of a small argon cluster embedded in a shell of helium atoms induced by soft X-ray ionization [28]. They found that the helium shell acts as an tamper due to the fast migration of electrons from the tamper shell to the highly-charged core. As a low first ionization potential is crucial for electron migration, hydrogen turns out to be much more efficient a tamper material than the isoelectronic helium which was previously discussed in this context [29].

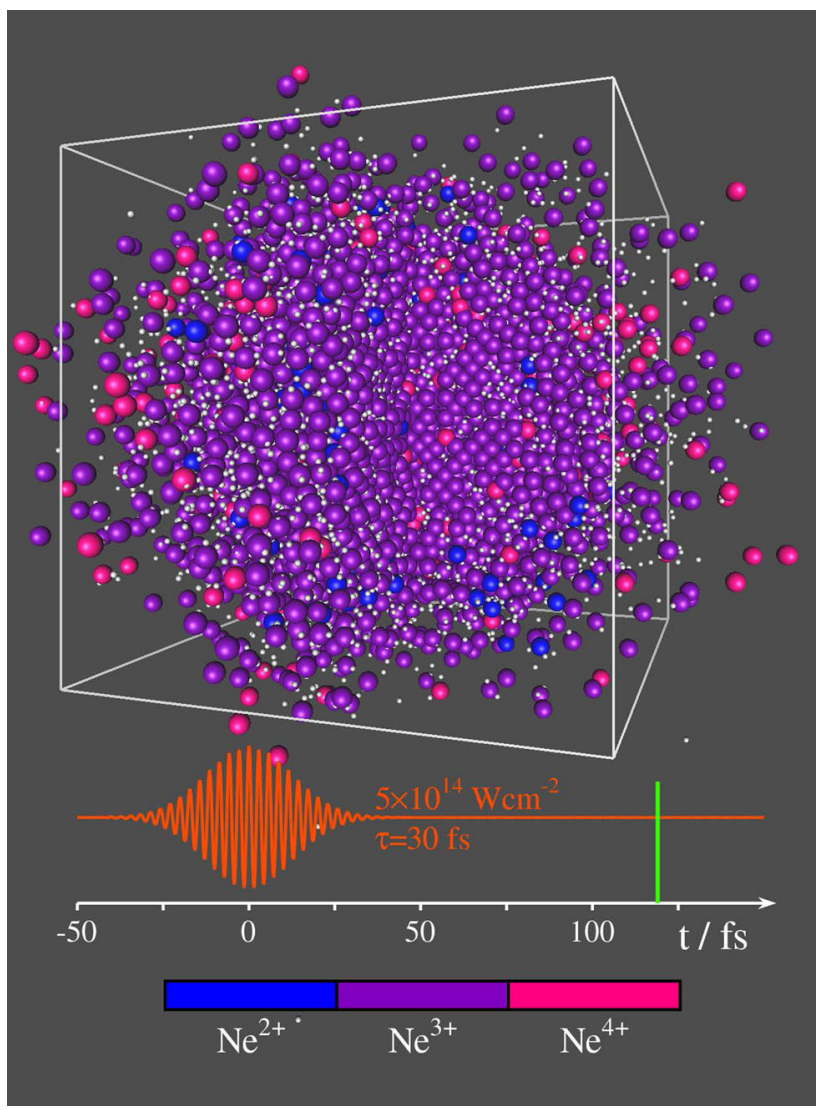

Fig. 1 Snapshot of an exploding $\mathrm{Ne}_{10149}$ cluster after irradiation with a short near-infrared laser pulse (pulse peak intensity $5 \times 10^{14} \mathrm{Wcm}^{-2}$, FWHM of the Gaussian electric field envelope $30 \mathrm{fs}$ ), obtained from a MD simulation. Ion charges are color coded, electrons are represented as small white spheres. A quarter of the atoms is cut out to allow for the view in the cluster interior. The time instant of the snapshot is represented as a green bar on the time axis together with the oscillating laser electric field. The white cube has a side length of $120 \AA$ and is added as a size comparison. Details of the MD simulation are given in the article by Last et al. in this issue [27]. By courtesy of A. Heidenreich

\section{High-order harmonic generation}

High-harmonic generation (HHG) in gases is a well established technique to generate ultrashort pulses of extreme ultraviolet up to X-ray radiation. Applications range from transient absorption spectroscopy of liquids and solids [30] to attosecond science [10]. However, the conversion efficiency of HHG, usually performed in atomic gases, is notoriously low $\left(<10^{5}\right)$. Therefore, condensed-phase targets are being explored in view of enhancing the yield and photon energy of the generated radiation. In particular, solid and nanostructured materials hold the promise of enhancing the HHG efficiency and extending the harmonic spectrum beyond the energy cut-offs limiting the achievable photon energy in gasphase HHG. 
In this special issue, Luu and Wörner have experimentally demonstrated that using standard, amplified 30-fs-laser pulses and quartz as a target material, HHG yields high photon energies [31]. By polarizing the input pulses circularly, circularly polarized XUV pulses were generated on demand. The XUV pulses had perfect degree of circularity within the uncertainty of the calibration measurement. These results are expected to open an extremely simple and effective way of generating circularly polarized XUV light for various applications such as circular dichroism experiments.

Yusofsani and Kolesik [32] have theoretically investigated the similarities in the response of metallic nanotips and nano-structures to free atoms driven by strong electric fields. To this end, they have generalized the Fowler-Nordheim model for the electron emission from a metal surface under the influence of an external electric field. They found out that in terms of the induced dipole moment per atom on the surface, the response is roughly as strong or even stronger than that observed in noble gases like argon and xenon. The expected harmonic spectrum only slowly falls off with the harmonic order.

Bauer and co-workers studied theoretically the spectra of HHG by various condensed-phase systems in view of utilizing high-harmonic spectroscopy for unravelling electronic structures and dynamics in condensed matter. Specifically, they modelled finite, hexagonal nanoribbons such as graphene and hexagonal boron nitride, in armchair and zig-zag configuration [33]. Different boundaries given by the geometry of the system are known to cause completely different high-harmonic spectra compared to the bulk. The authors used a tightbinding approach [34] as well as a description of HHG in the bulk of solids by replacing the orbital energies and the continuum by electronic bands [33]. They found that characteristic features in the harmonic spectra, such as onset and cut-off of the interband-harmonics plateau, can be understood with the help of the band structure of the respective system. The results for the finite ribbons are in agreement with those found from simulations without the tight-binding approximation. Thus, tight-binding approaches capture the essential mechanisms underlying HHG up to the cutoff. As they are computationally much less demanding, the authors suggest to use them for investigating much larger systems.

\section{New materials and THz spectroscopy}

From the pioneering work of Purcell [35] it is evident that the strong coupling between light and matter can significantly alter fundamental interactions to the extent that even spontaneous emission rates can be controlled. Polaritons, quasi-particle hybrids of matter and light arising out of the strong coupling between the two provide a versatile platform to investigate both the physics and applications in this unique regime [36]. Optical cavities, where electromagnetic fields are spa- tially confined, present ideal candidates for realizing polaritons. Solid state fabrication enables the realization of geometries and strategies such as distributed Bragg reflectors between which materials of interest can be sandwiched leading to the realization of polaritons [37]. Kottilil and co-workers present the essential criteria, design principles and configurations for implementing polaritons in organic microcavities [38]. The key advantage of the organic platform is facile synthesis of these systems both for investigating the physics and for leveraging applications using these schemes.

Femtosecond laser pulses in the optical and near-IR regimes have enabled table-top terahertz pulses in a frequency range where many molecular systems have unique spectral signatures or fingerprints [39]. These $\mathrm{THz}$ pulses are generated from fs pulses with near petahertz oscillating frequencies. Two routes enable this: the first employs photoconductive antennas in which temporal gradients in the photocurrent of carriers generated in semiconducting materials like low-temperature grown gallium arsenide lead to the emission of $\mathrm{THz}$ pulses. Alternately, optical rectification of fs pulses in suitable crystals such as ZnTe or more complex media such as nematic liquid crystals [40] can be exploited to generate $\mathrm{THz}$ ps pulses. While bolometric detectors present a relatively convenient way to measure $\mathrm{THz}$ radiation, time-domain spectroscopy remains the go to solution where electro-optic sampling of the timevarying electric field of these pulses by cross correlation with fraction of the parent optical pulse enables $\mathrm{THz}$ pulse metrology. One of the articles presented in this context reviews the numerical methods employed to determine material thickness using $\mathrm{THz}$ time-domain spectroscopy [41]. This work is also important from the context of understanding coherent artefacts in $\mathrm{THz}$ metrology which can potentially obscure and mislead measurements if not properly accounted for. Table-top $\mathrm{THz}$ pulse generation and detection provides a unique probe to obtain insights into quantum materials where emergent phenomena find a fertile playground. The state of the art in this context is adequately reviewed by Bera et al. [42].

\section{Theoretical aspects of strong light-matter interaction}

Even for small atomic systems, the theoretical foundations of intense laser-matter interactions posed challenges which have motivated developments tailored to suit these extremely non-perturbative situations $[43,44]$. This has led to multiple approaches suited to tackle different aspects of experimentally observed features such as the peak structures in above-threshold ionization, the plateau of high-harmonic spectra from atoms and molecules and the measurements of timedelays as well as so-called tunneling times. In this issue, Madsen [45] reviews the associated developments from a heuristic perspective. 
One of the important successes of intense lasermatter interaction has been the arrival of attosecond pulses in the extreme ultraviolet and soft X-ray spectral regions. These have effected a revolution enabling the study of sub- and few-femtosecond physics. These are the natural timescales of electronic dynamics. As a consequence, much recent attention has been paid to measuring photoionization time-delays in atoms and molecules. Although this was foreseen decades ago by Wigner [15] who discerned the existence of a generic time-delay which is the derivative of the scattering phase shift with energy. These have been directly measured in systems ranging from atoms to the condensed phase, leading to much debate about interpretation of experimental results especially when they are compared and matched to theoretical estimates and predictions [46]. Deshmukh et al. present an instructive tutorial on the Eisenbud-Wigner-Smith time delay from a theoretical perspective [47].

Two articles are dedicated to analytical formulations related to intense laser acceleration of charged particles. This theme is paramount to the physics and application of intense laser-matter interaction science as its potential is tremendous: not only do these accelerators provide unprecedented field gradients for acceleration $[48,49]$. Salamin et al. present a formulation of exploiting the chirp of laser pulses as a means to gain advantages in particle acceleration [50]. Mishra and Sengupta present methods and a useful code to arrive at a solution of the Hartemann-Luhmann equation [51].

\section{Experimental techniques}

Developments in experimental techniques continue to drive this field of research all the way from the serendipitous invention of chirped pulse amplification $[2,3]$ to the current generation of free-electron lasers $[52,53]$. This is also true for advanced time-domain investigations both in the $\mathrm{THz}$ [39] as well as attosecond domains $[10,46]$. In the former case, $\mathrm{THz}$ time-domain spectroscopy [40] has matured to become a standard technique to study advanced materials and their applications. Attosecond spectroscopy and metrology have similarly pushed the envelope in investigating electronic dynamics in quantum systems posing challenges for both experiment and theory as new avenues continue to be explored. This issue hosts articles which carry valuable insights and information on these aspects. Mukherjee et al. [41] review an important aspect of timedomain spectroscopy with table-top $\mathrm{THz}$ pulses. Determining sample thickness and coherent artefacts which arise from sample geometry play a considerable role in interpreting signals resulting from electro-optic sampling measurements. This tutorial consolidates the principles and methods underlying this important aspect of these investigations.

Attosecond pulses and table-top HHG based sources for investigating atomic, molecular and condensed phase systems have two important aspects to their implementation: the first is a successful realization of reliable and user-friendly workstations and beamlines akin to synchrotron facilities. Next, techniques towards stable, repeatable and rugged delay lines for attosecond time-domain experiments are paramount in determining the success in each laboratory putting in considerable efforts in this direction. Krikunova's group at the extreme light infrastructure (ELI) beamlines facility have put together an impressive facility in Prague which is open to users. They showcase the end-station for atomic, molecular and optical sciences titled-a Multi-purpose station for Atomic, molecular and optical sciences and Coherent diffractive imaging-MAC [54]. Here, the HHG emission from both a commercial as well as an in-house built laser system can be employed to perform time-resolved experiments with continuously replenished jet targets which could be atoms or molecules in supersonic jets, clusters pristine as well as doped-jets, substrate-free nanoparticles produced in an electrospray or a gas dynamics virtual nozzle focused using an aerodynamic lens to improve target density. This advanced end-station offers state-of-the-art diagnostics for electron - as well as ion-spectrometry - velocity-map imaging and timeof-flight spectrometers, respectively, and an X-ray camera. The authors who spiritedly push the envelope with additions and improvements to these targets and diagnostics intend to add a magnetic bottle spectrometer for detection and cryogenic continuous as well as pulsed nozzles to generate doped helium nanodroplets. This article may prove to be a valuable resource to current and forthcoming users as it showcases the technical capabilities at this impressive facility. On a different note, in the $\mathrm{THz}$ context, Mandal et al. present a tutorial on the various techniques involved in producing attosecond delays well-suited for pump-probe studies involving an attosecond pulse synchronized with infrared, optical, ultraviolet or extreme ultraviolet pulses [55]. Techniques for metrology, spectroscopy and time-resolved studies both in the attosecond and $\mathrm{THz}$ domains are well represented in this collection.

\section{Conclusion}

In this special issue, we bring together a representative collection of articles which span the entire range from the theoretical foundations of the physics of intense laser-matter interaction to applications in highharmonic generation and attosecond science, laserplasma acceleration, inducing highly ionized nanoplasmas and associated avenues such as strong-coupling leading to polaritons and terahertz pulses for spectroscopy. This field of research continues to burgeon as this collection was put together advancing some of the aspects covered here, e. $g$., zeptosecond delays in molecular photoionization [56]. This area of research will continue to leverage the opportunities provided by table-top femtosecond lasers in the optical and infrared domains owing to the Nobel-prize winning invention of 
chirped pulse amplification. It is very appropriate here to draw attention to rapid advancements in parallel, especially those in modern free-electron laser sources in the X-ray domain. These pave way to complementary approaches where angstrom spatial resolution and sub/few-fs temporal resolution come together along with electronic spectroscopy to provide a multi-dimensional view of matter and material systems ranging from atoms and molecules to clusters and micro-scale biological systems $[52,53]$. This is ample testimony to a bright future for this avenue of science which lights up physics, chemistry and biology, and indeed, heralds a multidisciplinary approach in both photon science and its applications. Thus, this issue is an intermittent milestone on the road and roadmap of the science, application and technology of intense laser-matter interactions.

Acknowledgements The authors gratefully acknowledge financial support by the Scheme for Promotion of Academic and Research Collaboration (SPARC), established by the Ministry of Human Resource Development, Government of India and by the International Network Programme of the Danish Agency for Science and Higher Education, the Ministry of Higher Education and Science, Denmark. We also acknowledge the support of the workgroups Quantum Center for Diamond and Emerging Materials (QuCenDiEM) as well as Micro-Nano-Bio-Fluidics funded by the Institute of Excellence (IoE) of the Ministry of Education, Govt. of India.

\section{References}

1. S. Haroche, Nobel lecture: Controlling photons in a box and exploring the quantum to classical boundary. Rev. Mod. Phys. 85(3), 1083 (2013)

2. G. Mourou, Nobel lecture: Extreme light physics and application. Rev. Mod. Phys. 91(3), 030501 (2019)

3. D. Strickland, Nobel lecture: Generating high-intensity ultrashort optical pulses. Rev. Mod. Phys. 91(3), 030502 (2019)

4. S.V. Bulanov, T. Esirkepov, T. Tajima, Light intensification towards the schwinger limit. Phys. Rev. Lett. 91(8), 085001 (2003)

5. P.W. Anderson, Physics: The opening to complexity. Proc. Natl. Acad. Sci. U.S.A. 92(15), 6653 (1995)

6. National Research Council et al. Controlling the quantum world: the science of atoms, molecules, and photons, volume 2. National Academies Press (2007)

7. M. Schirber, Nobel prize-lasers as tools. Physics 11, 100 (2018)

8. A.M. Zheltikov, Let there be white light: Supercontinuum generation by ultrashort laser pulses. PhysicsUspekhi 49(6), 605 (2006)

9. S.R. Fairchild, Y. Liu, J. Palastro, J. Peñano, Laser filamentation and applications: introduction. JOSA B 36(10), LFA1-LFA2 (2019)

10. F. Calegari, G. Sansone, S. Stagira, C. Vozzi, M. Nisoli, Advances in attosecond science. J. Phys. B Atom. Mol. Opt. Phys. 49(6), 062001 (2016)
11. F. Nan, Y. Liu, X. Ma, Z. Chen, Euv lithography: stateof-the-art review. J. Microelectron. Manuf 2, 1 (2019)

12. K.F. Kumar Pallav, E. Ishan Saxena, Laser-induced plasma micromachining process: Principles and performance. J. Micro Nano-Manufac. 3, 3 (2015)

13. J.M. Cole, D.R. Symes, N.C. Lopes, J.C. Wood, K. Poder, S. Alatabi, S.W. Botchway, P.S. Foster, S. Gratton, S. Johnson et al., High-resolution $\mu$ ct of a mouse embryo using a compact laser-driven X-ray betatron source. Proc. Natl. Acad. Sci. 115(25), 6335-6340 (2018)

14. V. Malka, S. Fritzler, E. Lefebvre, M.-M. Aleonard, F. Burgy, J.-P. Chambaret, J.-F. Chemin, K. Krushelnick, G. Malka, S.P.D. Mangles et al., Electron acceleration by a wake field forced by an intense ultrashort laser pulse. Science 298(5598), 1596-1600 (2002)

15. E.P. Wigner, Lower limit for the energy derivative of the scattering phase shift. Phys. Rev. 98(1), 145 (1955)

16. U. Saalmann, Ch. Siedschlag, J.M. Rost, Mechanisms of cluster ionization in strong laser pulses. J. Phys. B 39(4), R39 (2006)

17. T. Fennel, K.-H. Meiwes-Broer, J. Tiggesbäumker, P.G. Reinhard, P.M. Dinh, E. Suraud, Laser-driven nonlinear cluster dynamics. Rev. Mod. Phys. 82, 1793-1842 (2010)

18. T. Ditmire, T. Donnelly, A.M. Rubenchik, R.W. Falcone, M.D. Perry, Interaction of intense laser pulses with atomic clusters. Phys. Rev. A 53(5), 3379 (1996)

19. S.R. Krishnan, L. Fechner, M. Kremer, V. Sharma, B. Fischer, N. Camus, J. Jha, M. Krishnamurthy, T. Pfeifer, R. Moshammer et al., Dopant-induced ignition of helium nanodroplets in intense few-cycle laser pulses. Phys. Rev. Lett. 107(17), 173402 (2011)

20. S.R. Krishnan, Ch. Peltz, L. Fechner, V. Sharma, M. Kremer, B. Fischer, N. Camus, T. Pfeifer, J. Jha, M. Krishnamurthy, C.-D. Schröter, J. Ullrich, F. Stienkemeier, R. Moshammer, T. Fennel, M. Mudrich, Evolution of dopant-induced helium nanoplasmas. New J. Phys. 14(7), 075016 (2012)

21. A. Heidenreich, B. Grüner, M. Rometsch, S.R. Krishnan, F. Stienkemeier, M. Mudrich, Efficiency of dopantinduced ignition of helium nanoplasmas. New J. Phys. 18(7), 073046 (2016)

22. C. Medina, D. Schomas, N. Rendler, M. Debatin, D. Uhl, A. Ngai, L. Ben Ltaief, M. Dumergue, Z. Filus, B. Farkas et al., Single-shot electron imaging of dopantinduced nanoplasmas. New J. Phys. 23(5), 053011 (2021)

23. K.H. Meiwes-Broer, D. Komar, L. Kazak, J. Tiggesbäumker, Development of ion recoil energy distributions in the coulomb explosion of argon clusters resolved by charge-state selective ion energy spectroscopy. Eur. Phys. J. Special Topics (2021). https:// doi.org/10.1140/epjs/s11734-021-00108-x

24. J. Jha, R. Rajeev, T. Madhu Trivikram, M. Ram Gopal, A non-uniform charging scheme to decipher charge state propensities measured in nano-cluster ionization. Eur. Phys. J. Special Topics. (2021). https://doi.org/10. 1140/epjs/s11734-021-00027-x

25. C.A. Saladrigas, A.J. Feinberg, M.P. Ziemkiewicz, C. Bacellar, M. Bucher, C. Bernando, S. Carron, A.S. Chatterley, F.-J. Decker, K.R. Ferguson, L. Gomez, T. Gorkhover, N.A. Helvy, C.F. Jones, J.J. Kwok, A. Lut- 
man, D. Rupp, M.P. Rico, T.M. Tanyag, D.M. Neumark, C. Bostedt, A.F. Vilesov, O. Gessner, Charging and ion ejection dynamics of large helium nanodroplets exposed to intense femtosecond soft X-ray pulses. Eur. Phys. J. Special Topics 2, 2 (2021). https://doi.org/10. 1140/epjs/s11734-021-00280-0

26. S.S. Mahalik, M. Kundu, On the resonance absorption in laser-driven deuterium cluster. Eur. Phys. J. Special Topics 2, 2 (2021). https://doi.org/10.1140/epjs/ s11734-021-00029-9

27. I. Last, J. Jortner, A. Heidenreich, Two-directional collisional energy exchange between electrons and ions in exploding clusters. Eur. Phys. J. Special Topics 2, 2 (2021). https://doi.org/10.1140/epjs/ s11734-021-00107-y

28. A. Heidenreich, M. Mudrich, Efficient tampering of a coulomb exploding cluster embedded in a hydrogen shell. Eur. Phys. J. Special Topics 2, 2 (2021). https:// doi.org/10.1140/epjs/s11734-021-00190-1

29. C. Gnodtke, U. Saalmann, J.M. Rost, Ionization and charge migration through strong internal fields in clusters exposed to intense x-ray pulses. Phys. Rev. A 79(4), 041201 (2009)

30. R. Geneaux, H.J.B. Marroux, A. Guggenmos, D.M. Neumark, S.R. Leone, Transient absorption spectroscopy using high harmonic generation: a review of ultrafast x-ray dynamics in molecules and solids. Philos. Trans. R. Soc. A 377(2145), 20170463 (2019)

31. T.T. Luu, H.J. Wörner, Generation of circularly polarized extreme-ultraviolet harmonics from solids. Eur. Phys. J. Special Topics 2, 2 (2021). https://doi.org/10. 1140/epjs/s11734-021-00278-8

32. S. Yusofsani, M. Kolesik, Beyond fowler-nordheim model: harmonic generation from metallic nanostructures. Eur. Phys. J. Special Topics 2, 2 (2021). https://doi.org/10.1140/epjs/s11734-021-00189-8

33. H. Drüeke, D. Bauer, High-harmonic spectra of hexagonal nanoribbons from real-space time-dependent schrödinger calculations. Eur. Phys. J. Special Topics 2, 2 (2021). https://doi.org/10.1140/epjs/ s11734-021-00188-9

34. C. Jürß, D. Bauer, High-order harmonic generation in hexagonal nanoribbons. Eur. Phys. J. Special Topics. (2021). https://doi.org/10.1140/epjs/ s11734-021-00106-z

35. E. M. Purcell, Spontaneous emission probabilities at radio frequencies, in Confined Electrons and Photons (Springer, 1995), pp. 839-839

36. D.L. Mills, E. Burstein, Polaritons: the electromagnetic modes of media. Rep. Progr. Phys. 37(7), 817 (1974)

37. M. Nakayama, S. Komura, T. Kawase, D.G. Kim, Observation of exciton polaritons in a zno microcavity with hfo2/sio2 distributed bragg reflectors. J. Phys. Soc. Japan 77(9), 093705 (2008)

38. D. Kottilil, A. Babusenan, C. Vijayan, W. Ji, Strong light-matter interaction in organic microcavity polaritons: essential criteria, design principles and typical configurations. Eur. Phys. J. Special Topics 2, 2 (2021). https://doi.org/10.1140/epjs/s11734-021-00224-8

39. J.B. Baxter, G.W. Guglietta, Terahertz spectroscopy. Anal. Chem. 83(12), 4342-4368 (2011)
40. L. Cattaneo, M. Savoini, I. Muševič, A. Kimel, T. Rasing, Ultrafast all-optical response of a nematic liquid crystal. Optics Exp. 23(11), 14010-14017 (2015)

41. N.M. Soumya Mukherjee, P.C. Kumar, N.K. Upadhya, A review on numerical methods for thickness determination in terahertz time-domain spectroscopy. Eur. Phys. J. Special Topics 2, 2 (2021). https://doi.org/10.1140/ epjs/s11734-021-00215-9

42. A. Bera, S. Bera, Review of recent progress on thz spectroscopy of quantum materials: superconductors, magnetic and topological materials. Eur. Phys. J. Special Topics 2, 2 (2021). https://doi.org/10.1140/epjs/ s11734-021-00216-8

43. S.V. Popruzhenko, Keldysh theory of strong field ionization: History, applications, difficulties and perspectives. J. Phys. B Atomic Mol. Opt. Phys. 47(20), 204001 (2014)

44. P.W. Milonni, J.R. Ackerhalt, Keldysh approximation, a 2, and strong-field ionization. Phys. Rev. A 39(3), 1139 (1989)

45. Lars Bojer Madsen, Different forms of laser-matter interaction operators and expansion in adiabatic states. Eur. Phys. J. Special Topics. (2021). https://doi.org/10. 1140/epjs/s11734-021-00026-y

46. M. Hentschel, R. Kienberger, Ch. Spielmann, G.A. Reider, N. Milosevic, T. Brabec, P. Corkum, U. Heinzmann, M. Drescher, F. Krausz, Attosecond metrology. Nature 414(6863), 509-513 (2001)

47. P.C. Deshmukh, S. Banerjee, A. Mandal, S.T. Manson, Eisenbud-Wigner-Smith time delay in atom-laser interactions. Eur. Phys. J. Special Topics. 2, 2 (2021). https://doi.org/10.1140/epjs/s11734-021-00225-7

48. T. Tajima, K. Nakajima, G. Mourou, Laser acceleration. La Rivista del Nuovo Cimento 40(2), 33-133 (2017)

49. E. Esarey, C.B. Schroeder, W.P. Leemans, Physics of laser-driven plasma-based electron accelerators. Rev. Modern Phys. 81(3), 1229 (2009)

50. Y.I. Salamin, H.B. Benaoum, N.M. Jisrawi, Electron acceleration by a binomially chirped laser pulse. Eur. Phys. J. Special Topics. 2, 2 (2021). https://doi.org/10. 1140/epjs/s11734-021-00028-w

51. S.K. Mishra, S. Sengupta, Exact solution of hartemannluhmann equation of motion for a charged particle interacting with an intense electromagnetic wave/pulse. Eur. Phys. J. Special Topics 2, 2 (2021). https://doi.org/10. 1140/epjs/s11734-021-00260-4

52. C. Feng, H.-X. Deng, Review of fully coherent freeelectron lasers. Nucl. Sci. Tech. 29(11), 1-15 (2018)

53. H.N. Chapman, X-ray free-electron lasers for the structure and dynamics of macromolecules. Ann. Rev. Biochem. 88, 35-58 (2019)

54. E. Klimešová, O. Kulyk, Z. Hoque, A.H. Roos, K.P. Khakurel, M. Rebarz, M. Jurkovič, M. Albrecht, O. Finke, R. Lera, O. Hort, D.-D. Mai, J. Nejdl, M. Sokol, R.B. Fink, L.B. Ltaief, D. Westphal, A. Wolf, T. Laštovička, F. Frassetto, L. Poletto, J. Andreasson, M. Krikunova, A multipurpose end-station for atomic, molecular and optical sciences and coherent diffractive imaging at eli beamlines. Eur. Phys. J. Special Topics. 2, 2 (2021). https://doi.org/10.1140/epjs/ s11734-021-00192-z

55. A. Mandal, M.S. Sidhu, J.M. Rost, T. Pfeifer, K.P. Singh, Attosecond delay lines: design, char- 
acterization and applications. Eur. Phys. J. Special Topics. 2, 2 (2021). https://doi.org/10.1140/epjs/ s11734-021-00261-3
56. S. Grundmann, D. Trabert, K. Fehre, N. Strenger, A. Pier, L. Kaiser, M. Kircher, M. Weller, S. Eckart, L.P.H. Schmidt et al., Zeptosecond birth time delay in molecular photoionization. Science 370(6514), 339-341 (2020) 